\section{A Case of SAPHO Syndrome Presenting as Isolated Iliitis and Spondylitis}

To the Editor:

Synovitis, acne, pustulosis, hyperostosis, and osteitis (SAPHO) syndrome has a variable presentation and it is now believed that a number of previously described entities are cases of SAPHO. These include chronic recurrent multifocal osteomyelitis (CRMO) and pustulotic arthro-osteitis ${ }^{1}$. We describe a case of SAPHO presenting with iliitis and pyoderma gangrenosum as the sole manifestations.

A 39-year-old man was reviewed in the rheumatology outpatients department. He gave a history of episodic pain in both hips and his lower back since the age of 18 years. He had previously been diagnosed with HLA-B27-negative spondyloarthropathy at another center and had had a left hip replacement at the age of 22 years for secondary left hip osteoarthritis, and subsequently a revision of this at the age of 36 years when he fractured his left femur in a fall. He had developed a pyoderma gangrenosum at age 38 years. His clinical history and laboratory, radiological, and pathological investigations were reviewed. He had had persistently elevated inflammatory markers over the years, his latest erythrocyte sedimentation rate was $76 \mathrm{~mm} / \mathrm{h}$, C-reactive protein $73 \mathrm{mg} / \mathrm{l}$, hemoglobin 10.2 $\mathrm{g} / \mathrm{dl}$, and platelets $596 \times 10^{9} / 1$. Serum protein electrophoresis revealed a polyclonal gammopathy. Alkaline phosphatase was mildly elevated at 161 IU/1; the remainder of his biochemistry and hematology was normal. Plain radiography revealed severe chronic sclerotic change in the left hemipelvis (Figure 1). An isotope bone scan demonstrated increased activity in the left hemipelvis. Two previous bone biopsies had been reported as showing nonspecific focal inflammatory changes with negative cultures.

A provisional diagnosis of SAPHO syndrome was made. Computed tomography (CT) and magnetic resonance imaging (MRI) scans and a repeat bone biopsy of the left sacrum were performed in light of the clinical suspicion. CT (Figure 2) and MRI demonstrated marked osteitis in the left hemipelvis and sacral spine, with involvement extending to the L5 vertebra. The sacral biopsy demonstrated changes consistent with osteomyelitis. Microscopy and cultures including TB cultures and cultures for Propionibacterium acnes were negative. This confirmed the diagnosis of SAPHO. Treatment with nonsteroidal antiinflammatory drugs (NSAID) and intravenous pamidronate was commenced, with marked clinical improvement.

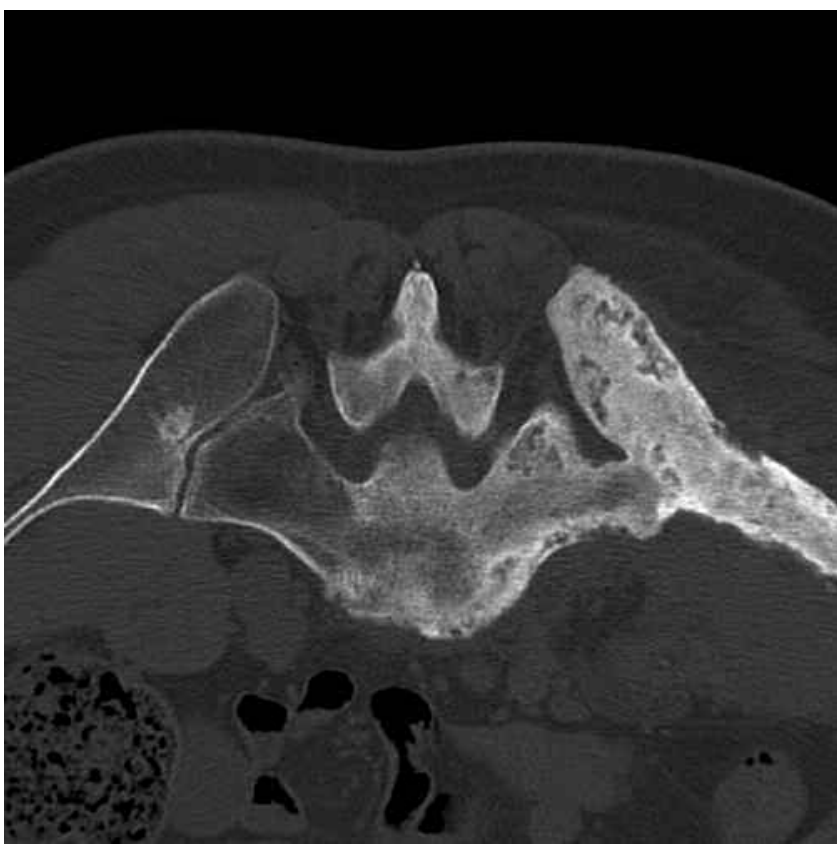

Figure 2. Computed tomography scan of the lumbosacral junction at the level of the S1 vertebra shows marked mixed sclerotic and lucent, almost "moth-eaten" appearance of S1 vertebra, adjacent ilium, and the posterior elements of L5. In addition there is fusion of the sacroiliac joint.

The initial diagnostic criteria proposed in 1987 are still used for SAPHO syndrome. The inclusion criteria comprise osteoarticular manifestations of severe acne or palmoplantar pustulosis; hyperostosis of the anterior chest wall, limb, or spine; or chronic recurrent multifocal osteomyelitis. Exclusion criteria include infectious osteomyelitis or arthritis (except P. acnes), diffuse idiopathic skeletal hyperostosis (DISH), palmoplantar keratoderma, and osteoarticular manifestations of retinoid therapy $^{2,3}$. The differential diagnosis of SAPHO is shown in Table 1.

SAPHO is at present considered a rare disease but the true prevalence

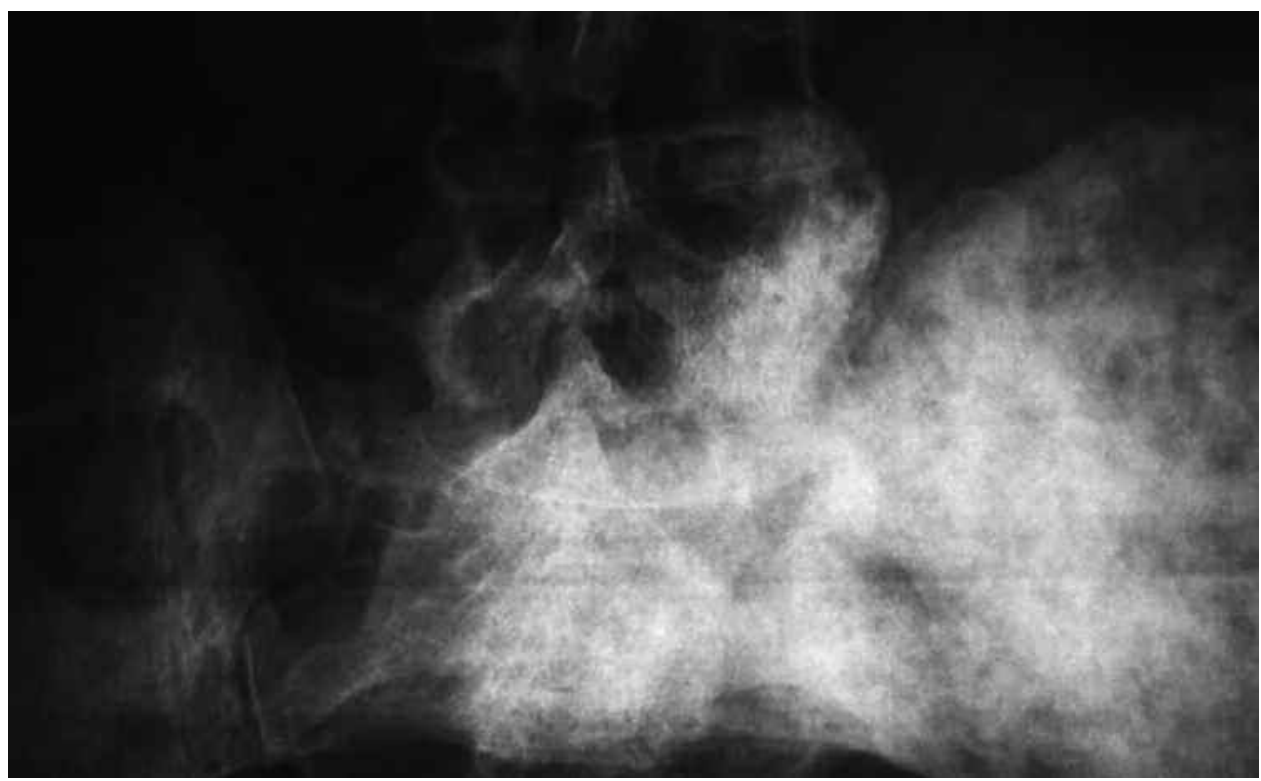

Figure 1. Focused radiograph of the left sacroiliac region shows marked patchy sclerosis with intermixed lucency of the left ilium and adjacent lower sacrum.

Personal non-commercial use only. The Journal of Rheumatology Copyright $@$ $~ 2011$. All rights reserved. 
SAPHO syndrome

Synovitis, acne, pustulosis, hyperostosis, osteitis. Not all patients have all features and some may have only one

Chronic recurrent multifocal In pediatrics often diagnosed as a unique entity, in adults classed as part of the SAPHO spectrum osteomyelitis

Pustulotic arthro-osteitis

PAPA (pyogenic arthritis, pyoderma gangrenosum, acne) syndrome

Psoriatic arthritis

Enteropathic arthritis
Now considered synonymous with SAPHO

Autosomal dominant disease. Causes non-axial sterile pyogenic arthritis followed by joint ankylosis

Osteitis mainly adjacent to entheseal sites. Some experts believe SAPHO to be a variant of psoriatic arthritis

Osteitis can occur in a similar pattern to psoriatic arthritis. Pyoderma gangrenosum and erythema nodosum can occur is probably underestimated. SAPHO has a female predominance ${ }^{4}$. Clinical presentation is dependent on the features present in a particular case. Bony lesions cause significant pain, with associated swelling at superficial sites. The anterior chest wall is the most common site, followed by the spine, but they can occur at virtually any bony site. Classic skin lesions include acne fulminans, hidradenitis suppurativa, and palmoplantar pustulosis. Less commonly described dermatological manifestations include pyoderma gangrenosum ${ }^{5}$, as in our case, and Sweet's syndrome. An association with inflammatory bowel disease has been reported in some series ${ }^{6}$. The clinical course is commonly marked by exacerbations and remissions; however, there may also be ongoing low-grade features throughout the course, as in our case.

Acute-phase reactants may be elevated, as in our case, but sometimes are not $^{4}$. An association with HLA-B27 has been suggested; however, recent case series ${ }^{4}$ indicate it to be no more frequent than in the general population.

Plain radiography demonstrates osteolysis in early cases, progressing to sclerotic changes in advanced disease. In very early disease, plain radiographs can be normal. Isotope bone scans demonstrate increased uptake in areas of active disease. SAPHO often has a characteristic appearance on MRI, with high signal on fat-saturated T2 fast spin-echo sequences and low signal on T1-weighted imaging, which enhances with gadolinium ${ }^{7}$, as in our case.

Bone biopsy is performed mainly to exclude infection and malignancy. There are no pathognomonic features of SAPHO on biopsy. Characteristically early lesions show inflammatory infiltrate with polymorphonuclear cells progressing to an infiltrate composed predominantly of lymphocytes and plasma cells. Noncaseating granulomas and multinucleate giant cells can also be seen. Chronic lesions may demonstrate fibrosis and new bone formation ${ }^{8}$. P. acnes has rarely been found at biopsy.

The treatment of SAPHO is largely based on case studies and expert opinion due to the rarity of the condition. NSAID are the first-line treatment employed in almost all patients. Steroids are usually effective in the short term, but can be associated with relapses following treatment withdrawal. Bisphosphonates, in particular pamidronate, appear to be particularly effective in the treatment of resistant cases of $\mathrm{SAPHO}^{9}$. The use of macrolide antibiotics such as azithromycin has been reported; they are thought to exert their effects through immunomodulatory rather than antibacterial mechanisms. Traditional disease-modifying antirheumatic drugs, particularly sulfasalazine and methotrexate, have a role in refractory cases. There have been reports of responses to anti-tumor necrosis factor agents ${ }^{10}$.

Our case demonstrates the need for awareness of atypical presentations of SAPHO syndrome, the potentially destructive course of untreated disease, and the dramatic response to targeted treatment once the diagnosis is made.

RICHARD CONWAY, MD, Rheumatology Specialist Registrar; ANTHONY RYAN, MD, Consultant Radiologist; KHALID KHAN, MD, Consultant Rheumatologist, Departments of Rheumatology and Radiology, Waterford Regional Hospital, Dunmore Road, Waterford, Ireland. Address correspondence to Dr. Conway;

E-mail: drrichardconway@gmail.com

\section{REFERENCES}

1. Sonozaki H, Mitsui H, Miyanaga Y, Okitsu K, Igarashi M, Hayashi Y, et al. Clinical features of 53 cases with pustulotic arthro-osteitis. Ann Rheum Dis 1981;40:547-53.

2. Chamot AM, Benhamou CL, Kahn MF, Beraneck L, Kaplan G, Prost A. Acne pustulosis hyperostosis osteitis syndrome. Results of a national survey. 85 cases. Rev Rhum Mal Osteoartic 1987;54:187-96.

3. Benhamou CL, Chamot AM, Khan MF. Synovitis acne pustulosis hyperostosis osteomyelitis syndrome (SAPHO): a new syndrome among the spondyloarthropathies? Clin Exp Rheumatol 1988;6:109-12.

4. Colina M, Govoni M, Orzincolo C, Trotta F. Clinical and radiologic evolution of synovitis, acne, pustulosis, hyperostosis, and osteitis syndrome: a single center study of a cohort of 71 subjects. Arthritis Rheum 2009;61:813-21.

5. Yamasaki O, Iwatsuky K, Kaneco F. A case of SAPHO syndrome with pyoderma gangrenosum and inflammatory bowel disease masquerading as Behcet's disease. Adv Exp Med Biol 2003;528:339-41.

6. Kahn MF, Bouchon JP, Chamot AM, Palazzo E. Chronic enterocolopathies and SAPHO syndrome. 8 cases. Rev Rhum Mal Osteoartic 1992;59:91-4.

7. Kirchhoff T, Merkesdal S, Rosenthal H, Prokop M, Chavan A, Wagner A, et al. Diagnostic management of patients with SAPHO syndrome: use of MR imaging to guide bone biopsy at CT for microbiological and histological work-up. Eur Radiol 2003;13:2304-8.

8. Girschick HJ, Huppertz HI, Harmsen D, Krauspe R, Müller-Hermelink HK, Papadopoulos T. Chronic recurrent multifocal osteomyelitis in children: diagnostic value of histopathology and microbial testing. Hum Pathol 1999;30:59-65.

9. Amital H, Applbaum YH, Aamar S, Daniel N, Rubinow A. SAPHO syndrome treated with pamidronate: an open-label study of 10 patients. Rheumatology 2004;43:658-61.

10. Massara A, Cavazzini PL, Trotta F. In SAPHO syndrome anti-TNF-alpha therapy may induce persistent amelioration of osteoarticular complaints, but may exacerbate cutaneous manifestations. Rheumatology 2006;45:730-3.

J Rheumatol 2011;38:8; doi:10.3899/jrheum.101372 Personal non-commercial use only. The Journal of Rheumatology Copyright @ 2011. All rights reserved. 